\title{
ECR-MAPK Regulation in Liver Early Development
}

\author{
Xiu-Ju Zhao ${ }^{1,2}$ and Hexian Zhuo ${ }^{3}$ \\ ${ }^{1}$ School of Biology and Pharmaceutical Engineering, Wuhan Polytechnic University, Collaborative Innovation Center of \\ Processing of Agricultural Products in Hubei Province, No. 68 South Xuefu Road, Changqing Garden, Wuhan 430023, China \\ ${ }^{2}$ Wuhan Institute of Physics and Mathematics, Chinese Academy of Sciences, West No. 30 Xiaohongshan, Wuhan 430071, China \\ ${ }^{3}$ Xinxiang Institute for Drug Control, No. 17 Jiankang Road, Xinxiang 453000, China \\ Correspondence should be addressed to Xiu-Ju Zhao; dzrdez@163.com
}

Received 6 June 2014; Revised 27 August 2014; Accepted 16 September 2014; Published 18 December 2014

Academic Editor: Klaus Wimmers

Copyright ( 2014 X.-J. Zhao and H. Zhuo. This is an open access article distributed under the Creative Commons Attribution License, which permits unrestricted use, distribution, and reproduction in any medium, provided the original work is properly cited.

Early growth is connected to a key link between embryonic development and aging. In this paper, liver gene expression profiles were assayed at postnatal day 22 and week 16 of age. Meanwhile another independent animal experiment and cell culture were carried out for validation. Significance analysis of microarrays, qPCR verification, drug induction/inhibition assays, and metabonomics indicated that alpha-2u globulin (extracellular region)-socs2 (-SH $\mathrm{S}_{2}$-containing signals/receptor tyrosine kinases)ppp2r2a/pik3c3 (MAPK signaling)-hsd3b5/cav2 (metabolism/organization) plays a vital role in early development. Taken together, early development of male rats is ECR and MAPK-mediated coordination of cancer-like growth and negative regulations. Our data represent the first comprehensive description of early individual development, which could be a valuable basis for understanding the functioning of the gene interaction network of infant development.

\section{Introduction}

Development is differential expression of the genome of organisms in different time points. Mammalian liver especially plays a vital role in the coordination of various physiological processes, and due to the different metabolic needs for male and female reproduction, mammalian liver shows considerable sexual dimorphism; this phenotypic expression is mediated via sex hormones [1]. Androgen response of the rat liver shows notable change during individual development and only the postpubertal adult (40-750 days of age) is subject to androgen-inducible genes and androgen-repressible genes [2]. Thus, transcriptional control in mammals must be properly coregulated for early stages of liver formation, perinatal repression, and position-dependent regulation [3]. Furthermore, expression profiles of fetal and natal liver tissues from mice reveal two stages during embryonic liver development; embryonic day 14.5 is a transition point when hepatocytes occur. Postnatal processes are also divided into two stages (III and IV) and genes expression profile of stage IV (ranging from day 7 to week 18) exhibited more invariant property [4].
Previous researches focus on embryonic development, using targeted methods such as genetic modification, quantitative PCR, hybridization, and electrophoresis. However, early growth is less concerned. Early growth consists of natal, prepuberty, puberty, and adult stages. And moreover, early growth is connected to a key link between embryonic development and aging [4]. Therefore it is necessary to deepen the study of the early development of individual growth and thus to provide a basis and reference for dietary intake and disease prevention and control in the process of human growth, especially infant.

Utilizing systems biology approaches, for example, by combining global gene expression profiling and metabolic pattern techniques, provides means to determine characteristic transcript profile and endpoint metabolic effects of development. Integrated information from transcriptomic and metabonomic profiling contributes to elucidate mechanisms of a developmental effect in detail and with comprehensiveness.

This research compared the gene expression profiles of 22 days ( 3 weeks) and 16 weeks of age, using Wistar rat as a model 
from public database, and furthermore clinical biochemistry, qPCR, cell culture, and NMR were carried out for validation and confirmation from independent animal experiment, to reveal temporal migration information and the transcription pattern of this early growth process.

\section{Materials and Methods}

2.1. Animal Experiment and Cell Culture. This study partially came from public database GSE32156 [5]. Briefly, offspring from Wistar Han dams were fed normally. Liver samples were collected at postnatal day (PND) $22(n=5)$ and week 16 $(n=5)$ of age for liver gene expression profile analysis. Independent animal experiment was carried out according to guidelines of the government of China. Sera for clinical biochemistry, urines for NMR, and livers for qPCR and cell culture were collected when the rats were decapitated after anesthesia with isoflurane. Rat primary liver cells were cultured and dexamethasone $(\operatorname{dex}, 0.1 \mu \mathrm{M})$ or cycloheximide $(\mathrm{CHX}, 0.05 \mu \mathrm{g} / \mathrm{mL})$ was added as indicated.

2.2. Clinical Biochemistry. Sera were analyzed for glucose (Glc), total cholesterol (CHOL), creatinine (CREA), triglyceride (TG), albumin (ALB), aspartate aminotransferase (AST), alanine aminotransferase (ALT), alkaline phosphatase $(\mathrm{AlkP})$, total protein (TP), and testosterone using biochemical analyzer and radioimmunoassay.

2.3. Transcriptomic Analysis. Total RNA was isolated from 10 rat livers, 5 from the control pups and 5 from the adults, with Trizol Reagent (Invitrogen Corp., Carlsbad, CA), in accordance with the manufacturer's instructions. The concentration and purity of total RNA were determined by spectrophotometer, $28 \mathrm{~S}$ and $18 \mathrm{~S}$ rRNA. The Affymetrix Rat Genome 2302.0 arrays were used to monitor variations in gene expression profile. The log-transformed (base $=2$ ) data were obtained for all probes and array-wise normalized using Affymetrix Dchip 2006.

The $t$-test and Wilcoxon signed-rank test were utilized for significance analysis of microarrays (SAM) [6-9]. A permutation test was employed for estimating the falsediscovery rate (FDR $<0.05, n=200 \sim 500)$. The CapitalBio Molecule Annotation System (MAS), KEGG, and GenMAPP databases were used for pathway analysis (http://bioinfo.capitalbio.com/mas). For each pathway, genes with known rat orthologues were compared with sets of significant genes from SAM to define the effects of corresponding pathway.

The relationship of genes or gene clusters was carried out using Pearson's correlation, Spearman's correlation, or 2D STOCSY (statistical total correlation spectroscopy).

2.4. Quantitative Real-Time PCR and Western Blot. cDNA was synthesized using an oligo-(dT)15 primer (Invitrogen). PCR primers were designed with Primer Premier 5.0 software. The housekeeping gene $\beta$-actin was used as an internal control. The PCR amplification was conducted at $95^{\circ} \mathrm{C}$ for $15 \mathrm{~min}$, followed by 40 cycles of $94^{\circ} \mathrm{C}$ for $5 \mathrm{~s}, 58^{\circ} \mathrm{C}$ for $15 \mathrm{~s}$, and $72^{\circ} \mathrm{C}$ for $10 \mathrm{~s}$. The relative mRNA levels of selected genes were calculated with the $2^{-\Delta \Delta \mathrm{Ct}}$ method [10]. Liver proteins were subjected to sodium dodecyl sulfate-polyacrylamide gel electrophoresis and transferred to blotting membrane. Immunoblots were blocked with $3 \%$ bovine serum albumin in Tris-buffered saline/Tween-20 buffer for $60 \mathrm{~min}$ at room temperature and incubated overnight at $4^{\circ} \mathrm{C}$ with primary antibodies. Blots were developed by an Enhanced Chemiluminescence Western blotting kit (Amersham Biosciences, Uppsala, Sweden) and visualized by a Gene Genome bioimaging system. Bands were analyzed by densitometry with GeneTools software (Syngene, Frederick, MD, USA). Values were reported as means \pm SD. Statistical differences were determined by the one-way ANOVA multiple range test and the Wilcoxon rank sum test. Statistical significance was set at $P<0.05$.

2.5. NMR Spectroscopy Acquirement. $550 \mu \mathrm{L}$ urine was mixed with $55 \mu \mathrm{L}$ of phosphate buffer, followed by centrifugation. 1D ${ }^{1} \mathrm{H}$ NMR spectra were acquired (298 K, Bruker Avance III-600 MHz NMR spectrometer) with 32 transients for urine using a standard presaturation pulse sequence (presaturation during a relaxation delay and during the mixing time). $2 \mathrm{D}$ J-resolved, ${ }^{1} \mathrm{H}-{ }^{1} \mathrm{H}$ correlation, total ${ }^{1} \mathrm{H}-{ }^{1} \mathrm{H}$ correlation, ${ }^{1} \mathrm{H}$ ${ }^{13} \mathrm{C}$ heteronuclear single quantum coherence, and ${ }^{1} \mathrm{H}_{-}{ }^{13} \mathrm{C}$ heteronuclear multiple bonds correlation NMR spectra [1113] were acquired for selected urine to assign metabolites.

2.6. Statistical Analysis of NMR Spectra. NMR spectra were processed routinely [14] for phase, baseline, and chemical shift reference calibrations.

Unsupervised PCA (principal component analysis) was performed (SIMCA-P 11.0 demo, Umetrics, Sweden) to outline intrinsic similarity/dissimilarity within the data set scaled to unit variance (UV). Comparisons between temporal animals were made by carrying out qualitative PLS (partial least square regression) and O-PLS (orthogonal projection to latent structures) models (class information as qualitative $Y$ variable). The validity of the models was assessed by $Q^{2}$ (predictability) and $R^{2}$ (interpretability) of the model. Meanwhile, the same models were validated by a 7 -fold cross validation [15], cross validation-ANOVA [16-18], and a permutation test $(n=200)$ [19]. Valid models including significantly changed metabolites (denoted by red color) were visualized and shown in the colored correlation coefficient loading plots (MATLAB version 7.1, Mathworks Inc; Natwick, USA).

\section{Results}

3.1. Weight and Clinical Biochemistry of Early Individual Development. Adult rats $(\sim 313.6 \mathrm{~g})$ have much more weight than pups $(\sim 219.3 \mathrm{~g})(P<0.001$, Table 1$)$. Serum clinical biochemistry data from the adult rats contained higher levels of metabolites, such as glucose, triglyceride, testosterone, and lower enzymes, such as aspartate aminotransferase (AST), alanine aminotransferase (ALT), and alkaline phosphatase 
TABLE 1: Body mass and biochemistry indices between adults and pups.

\begin{tabular}{lccc}
\hline Index & Pup & Adult & $P$ \\
\hline Body mass, g & $219.3 \pm 8.8$ & $313.6 \pm 20.8$ & $1.0 E-12$ \\
ALT, IU/L & $37.5 \pm 3.8$ & $30.8 \pm 3.9$ & 0.00041 \\
AST, IU/L & $176.7 \pm 22.2$ & $160.2 \pm 30.9$ & 0.035 \\
AlkP, IU/L & $70.7 \pm 12.9$ & $41.6 \pm 4.6$ & $5.7 E-07$ \\
Glc, mmol/L & $4.8 \pm 0.5$ & $5.5 \pm 0.4$ & 0.00035 \\
TG, mmol/L & $0.57 \pm 0.13$ & $0.76 \pm 0.26$ & 0.033 \\
Testosterone, & $25 \pm 3$ & $40 \pm 4$ & 0.046 \\
ng/mL & & &
\end{tabular}

(AlkP) compared with those from the pup rats $(P<0.05$, Table 1).

3.2. Coexpressing Genes of Early Individual Development. Coexpressing genes between adults and pups belong to REDOXIDATION [ATP binding, electron carrier activity, oxoglutarate dehydrogenase (succinyl-transferring) activity, L-2-hydroxyglutarate dehydrogenase], PROTEIN TRANSLATION, TRANSLOCATION AND PROTEOLYSIS [ADPribosylation factor binding, glutamyl-tRNA aminoacylation, proteolysis, protein tyrosine phosphatase activity, SMAD protein nuclear translocation], TRANSCRIPTION REGULATION [ADP-ribose diphosphatase nucleotide and nucleic acid metabolic process, $3^{\prime}$ pre-RNA cleavage], CELL CYCLE [nucleotide-excision repair (cyclin-dependent protein serine/threonine kinase activity), positive regulation of cell proliferation], and SYSTEM ORGANIZATION [acrosome intracellular phorbol ester receptor signaling cascade, nervous system development, motor axon guidance, neurotransmitter acetylcholine receptor activity, $G$ protein coupled olfactory receptor protein signaling pathway, detection of chemical stimulus involved in sensory perception of bitter taste, keratin filament, regulation of the force of heart contraction, regulation of hindgut contraction] (Supplementary Table $1, P>0.965,0.9999 \leqq$ change fold $\leqq 1.0001$ ) (see Supplementary Table 1 in Supplementary Material available online at http://dx.doi.org/10.1155/2014/850802).

3.3. Differential Genes of Early Individual Development. The differential genes between adults and pups were listed (Table 2), of which 48 genes/probe sets were upregulated $(P<$ 0.05 , fold $>1.53)$ and 30 genes were downregulated $(P<0.05$, fold $<0.665)$. According to GO terms [20], these BIOLOGICAL PROCESSES were transport, C21-steroid hormone biosynthetic process, metabolic process, oxidation reduction, estrogen metabolic process, digestion, cell morphogenesis, oxygen and reactive oxygen species metabolic process, superoxide metabolic process, transcription, cell proliferation, cell differentiation, protein amino acid dephosphorylation, regulation of cell growth, cell adhesion, immune response, and proteolysis; CELL COMPONENTS were extracellular region and space, mitochondrion, endoplasmic reticulum, peroxisome, integral to membrane, protein phosphatase type 2A complex, plasma membrane, and Golgi-associated vesicle;
MOLECULAR FUNCTIONS were transporter activity, catalytic activity, 3-beta-hydroxy-delta-5-steroid dehydrogenase activity, monooxygenase activity, endopeptidase inhibitor activity, estrone sulfotransferase activity, estradiol 17-betadehydrogenase activity, identical protein binding, glucuronosyltransferase activity, superoxide-generating NADPH oxidase activity, nucleic acid binding, methyltransferase activity, protein binding, protein tyrosine/serine/threonine phosphatase activity, oxidoreductase activity, protein phosphatase type $2 \mathrm{~A}$ regulator activity, growth hormone receptor binding, proton-dependent oligopeptide secondary active transmembrane transporter activity, metalloendopeptidase activity, sterol/transporter activity, asparagine synthase (glutaminehydrolyzing) activity, cytokine activity, transferase activity (transferring acyl groups other than aminoacyl groups), aldoketo reductase activity, and so forth.

3.4. Pathway Significance of Early Individual Development. The significant pathways were as follows: transport (obp3(AB039825), obp3(J00738), AB039826, AB039823, ust5r, mup5, AB039828, clca5, abcg8, abcd2, aqp7, and abcc3; $P=0.001)$ and cell adhesion (cdh17, omd, jam3, pcdh17, ncam2, amigo1; $P=0.005$ ); steroid biosynthesis (obp3, $h s d 3 b 5$, akrlc1, hsdl2, hsd17b6, hsd11b2, ar, and cyp17a1; $P=8.6 e-9)$, metabolic process (hsd3b5, dhrs7, hao2, mett17b, hsdl2, asns, acsm2, and psat1; $P=0.035$ ), oxidation reduction ( $h s d 3 b 5, c y p 2 c 13, c y p 3 a 9, c y p 2 a 2$, hao2, akrlc1, cyp2c29, nox4, rgd1564865, hsdl2, me3, cyp2c12, cyp17al, and akrlb7; $P=0.0001$ ), and fatty acid biosynthesis (scd, fasn; $P=0.00014)$; transcription regulation (zfp37, zfp 68, npas 2 , taf9b, ppargcla, and $n f e 2 ; P=0.0003$ ) and regulation of cell cycle (ccng2, ccnb2, and ccna2; $P=0.00018$ ); skeletal system development (col4a1, col5a2, col5a1, colla2, and colla1; $P=$ $4.4 e-5)$ and organization (cav2, pex11a, onecut1, meox2, $c m l 3$, col5a2, and lox; $P=0.0051)$; immune response (rt1-ce5, cxcl9, rt1-aw2, tnfsf13, and cxcl13; $P=0.099$ ), signaling (ppp2r2a, socs2, olr59, rgs3, adora2b, cish, atp6ap2, pik3c3, ppp1r2, ghr, and nrg1; $P=0.00067$ ), and proteolysis ( $m u p 5$, mme, trhde, spink3, prcp, and rgd1562284; $P=9.1 e-4)$ (Table 3).

3.5. Genes Correlation Network of Early Individual Development. Furthermore, obp3, extracellular region and transporter, was correlated to membrane (ust5r, stac3, cdh17, mme, olr59, gpm6a, tmem163, abcg8, abcd2, and abcc3), adapter (stac3, socs 2$)$, transcription ( $z f p 37, c c n a 2, a s n s$, and rgd1562284), immune (rt1-ce5, rt1-aw2, cxcl13, and cyfip2), and redox (hsd3b5, cyp2c13, cyp2a2, dhrs7, hao2, akr1c1, nox4, inmt, dusp1, mettl7b, ppp2r2a, cyp17a1, and akr1b7) $(|r|>$ $\left|r_{\text {cutoff }}\right|=0.632, P<0.05$ ) (Figure 1, Supplementary Table 2).

3.6. qPCR Validation of Early Individual Development. qPCR validation for highlighted microarray genes was carried out for pups and adults. The results demonstrated that the mRNA level of $o b p 3$, a major regulator in odorant binding, was elevated 3.75-fold in the adult group compared to pups; the expression levels of rup2, hsd3b5, dhrs7, cyp2c13, ust5r, stac3, 
TABLE 2: Significantly changed genes between 16 weeks and 3 weeks old.

\begin{tabular}{|c|c|c|c|}
\hline Public ID & Gene symbol & $P$ & Fold \\
\hline AB039825 & $o b p 3$ & $2.12 E-07$ & 3.75 \\
\hline J00738 & obp3 & $6.23 E-07$ & 3.45 \\
\hline AF368860 & $\begin{array}{c}\operatorname{loc} 680367 \\
/ / / \operatorname{loc} 680406 / / / \text { rup2 }\end{array}$ & $2.97 E-06$ & 3.40 \\
\hline AF198441 & rup2 & 0.000102 & 3.26 \\
\hline NM_012584 & $h s d 3 b 5$ & $2.02 E-08$ & 3.14 \\
\hline BI288203 & $d h r s 7$ & $3.84 E-06$ & 3.05 \\
\hline AF368860 & loc680367 /// rup2 & 0.000457 & 3.02 \\
\hline J02861 & cyp $2 c 13$ & $2.66 E-05$ & 2.93 \\
\hline AA893518 & loc680367 & 0.00028 & 2.87 \\
\hline AI043805 & & $3.83 E-06$ & 2.77 \\
\hline AB039826 & $\begin{array}{c}\operatorname{loc} 259245 \\
/ / / \operatorname{loc} 259246 / / / \text { mup5 }\end{array}$ & $8.02 E-07$ & 2.68 \\
\hline $\mathrm{U} 46118$ & cyp3a9 & $2.99 E-05$ & 2.52 \\
\hline АВ039823 & $\operatorname{loc} 259246$ & $3.95 E-07$ & 2.51 \\
\hline NM_012693 & cyp2a2 & $1 E-06$ & 2.45 \\
\hline NM_032082 & hao2 & $6.58 E-05$ & 2.36 \\
\hline NM_012883 & sult1e1 & $1.81 E-05$ & 2.34 \\
\hline NM_134380 & $u s t 5 r$ & $7 E-05$ & 2.20 \\
\hline AI072107 & $a k r 1 c 1$ & $9.61 E-05$ & 2.16 \\
\hline АВ039828 & mup5 & 0.000142 & 2.10 \\
\hline BM385735 & stac3 & $4.67 E-05$ & 2.07 \\
\hline NM_019184 & cyp2c29 & $4.09 E-05$ & 1.96 \\
\hline NM_053524 & nox4 & 0.000186 & 1.88 \\
\hline AF072439 & $z f p 37$ & $9.94 E-05$ & 1.75 \\
\hline AW523958 & & 0.001031 & 1.73 \\
\hline AI232716 & inmt & 0.000633 & 1.71 \\
\hline AW521319 & & $1.35 E-05$ & 1.70 \\
\hline BM390462 & rgd1310209 & $1.06 E-05$ & 1.68 \\
\hline BF417649 & & $5.11 E-06$ & 1.68 \\
\hline BI289963 & & $1.2 E-05$ & 1.67 \\
\hline AI454016 & $\operatorname{lrtm} 2$ & 0.002522 & 1.66 \\
\hline BE110108 & dusp1 & 0.002989 & 1.66 \\
\hline AA866264 & rgd1564865 & $7.97 E-05$ & 1.63 \\
\hline AI548958 & hrasls & 0.000412 & 1.63 \\
\hline AB039828 & тuр5 & 0.004619 & 1.63 \\
\hline AI071674 & & 0.000412 & 1.62 \\
\hline AA892888 & mettl7b & $3.29 E-06$ & 1.62 \\
\hline BF395080 & & 0.000385 & 1.61 \\
\hline AI136882 & rgd1560784 & 0.000154 & 1.60 \\
\hline NM_053999 & $p p p 2 r 2 a$ & $9.91 E-06$ & 1.60 \\
\hline NM_058208 & $\operatorname{socs} 2$ & 0.000126 & 1.60 \\
\hline BF289150 & & $5.23 E-05$ & 1.60 \\
\hline NM_053977 & $c d h 17$ & 0.027013 & 1.58 \\
\hline AW434139 & & $9.35 E-08$ & 1.58 \\
\hline
\end{tabular}

TABLE 2: Continued.

\begin{tabular}{|c|c|c|c|}
\hline Public ID & Gene symbol & $P$ & Fold \\
\hline NM_012608 & mme & 0.000567 & 1.57 \\
\hline BG375383 & rgd1308116 (nim1) & $3.05 E-05$ & 1.55 \\
\hline AI716500 & & 0.001038 & 1.54 \\
\hline BF414998 & $\begin{array}{l}\text { rgd1306105 } \\
(\text { tmem150c) }\end{array}$ & $9.72 E-05$ & 1.54 \\
\hline AJ243338 & rt1-ce5 & 0.090847 & 1.53 \\
\hline BF558981 & pcdh17 & 0.004396 & 0.67 \\
\hline NM_031572 & cyp2c12 & $6.21 E-05$ & 0.66 \\
\hline NM_130414 & $a b c g 8$ & 0.004836 & 0.66 \\
\hline NM_012753 & cyp17a1 & $2.67 E-05$ & 0.66 \\
\hline NM_033352 & $a b c d 2$ & 0.000309 & 0.66 \\
\hline AA998516 & ccna2 & 0.000703 & 0.66 \\
\hline U07202 & asns & 0.00178 & 0.65 \\
\hline BI281851 & loc685203 & 0.002673 & 0.64 \\
\hline NM_012488 & $a 2 m$ & 0.000111 & 0.64 \\
\hline NM_031050 & lum & 0.001029 & 0.64 \\
\hline NM_012521 & $s 100 g$ & 0.016704 & 0.64 \\
\hline AA892854 & cxcl13 & 0.002336 & 0.64 \\
\hline AF062389 & $\operatorname{acsm} 2$ & 0.000189 & 0.63 \\
\hline BE112927 & cyfip2 & 0.00012 & 0.63 \\
\hline AI101139 & tox & 0.000121 & 0.63 \\
\hline NM_019157 & $a q p 7$ & 0.000186 & 0.62 \\
\hline AI230228 & psat1 & $9.8 E-05$ & 0.62 \\
\hline AW523490 & & 0.001056 & 0.62 \\
\hline AI408151 & rgd1566215 & $2.5 E-05$ & 0.60 \\
\hline AW252129 & $n f e 2$ & 0.003234 & 0.60 \\
\hline BE116152 & elovl6 & $5.18 E-05$ & 0.59 \\
\hline BM390001 & rgd1562284 & 0.000166 & 0.59 \\
\hline AF072816 & $a b c c 3$ & 0.000377 & 0.58 \\
\hline AI235528 & & $4.13 E-05$ & 0.56 \\
\hline BF284168 & & $6.65 E-08$ & 0.54 \\
\hline BF396857 & elovl6 & 0.000111 & 0.53 \\
\hline AI599365 & & 0.00087 & 0.52 \\
\hline AA963228 & & 0.000112 & 0.48 \\
\hline AI102401 & & 0.000117 & 0.45 \\
\hline NM_053781 & $a k r 1 b 7$ & 0.000265 & 0.40 \\
\hline
\end{tabular}

$z f p 37, p p p 2 r 2 a, \operatorname{socs} 2$, atp6ap2, pik3c3, and $g h r$ were elevated significantly in the adult rats compared to the pups, while the expression levels of $p c d h 17, a b c g 8, c c n a 2, s 100 \mathrm{~g}, c x c l 13$, tox, and $a k r 1 b 7$ were decreased significantly (Figure 2(a), $P<$ $0.01)$.

3.7. Induction/Inhibition of obp3 and Its Targets. In order to characterize the interplay between $o p b 3$ and its potential targets, we evaluated the dexamethasone induction/cycloheximide inhibition of $o b p 3$ and coregulated genes of adults. Dexamethasone $(0.1 \mu \mathrm{M})$ induced $o b p 3$ expression and upregulated its targets: hsd $3 b 5$, socs 2 , pik3c3, 
TABLE 3: Significant regulated pathways between adults and pups.

\begin{tabular}{|c|c|c|}
\hline Pathway & Genes & $P$ \\
\hline Transport & obp3, mup5, ust5r, mme, clca5, gpm6a, tmem163; abcg8, abcd2, aqp7, abcc3 & 0.001 \\
\hline Steroid & obp3, hsd3b5, akr1c1, hsdl2, hsd17b6, hsd11b2, ar; cyp17al & $8.6 e-9$ \\
\hline Metabolic process & hsd3b5, dhrs7, hao2, mett17b, hsdl2; asns,acsm2,psat1 & 0.035 \\
\hline Redox & $\begin{array}{l}\text { hsd365, cyp2c13, cyp3a9, cyp2a2, hao2, akr1c1, cyp2c29, nox4, rgd1564865, hsdl2, } \\
\text { me3; cyp2c12, cyp17al, akr1b7 }\end{array}$ & 0.0001 \\
\hline Transcription regulation & $z f p 37, z f p 68$, npas2; taf $9 b$, ppargcla, $n f e 2$ & 0.0003 \\
\hline Cell adhesion & cdh17, omd, jam3; pcdh17, ncam2, amigo1 & 0.005 \\
\hline Collagen & ;col4a1, col5a2, col5a1, colla2, colla1 & $4.4 e-5$ \\
\hline Immune response & rt1-ce5, cxcl9, rt1-aw2, tnfsf13; cxcl13 & 0.099 \\
\hline Signaling & ppp2r2a, socs2, olr59, rgs3, adora2b, cish, atp6ap2, pik3c3, ppp1r2, ghr, nrg1; & 0.00067 \\
\hline Organization & cav2, pex11a, onecut1, meox2, cml3; col5a2, lox, & 0.0051 \\
\hline Fatty acid biosynthesis & $s c d$, fasn; & 0.00014 \\
\hline Cyclin & ccng2; ccnb2, ccna2 & 0.00018 \\
\hline Solute carrier (slc) & 41a2, 25a30, 9a3r1, 31a2; 13a3,1a3, 4a1, & 0.0033 \\
\hline Proteolysis (peptidase) & mup5, mme, trhde, spink3, prcp; rgd1562284 & $9.1 e-4$ \\
\hline Ubiquitin & $h s p b 1, u s p 18$ & 0.029 \\
\hline $\mathrm{G}$ protein & gnai3 gnat3 adora2b; & 0.0094 \\
\hline
\end{tabular}

Gene: higher; lower.

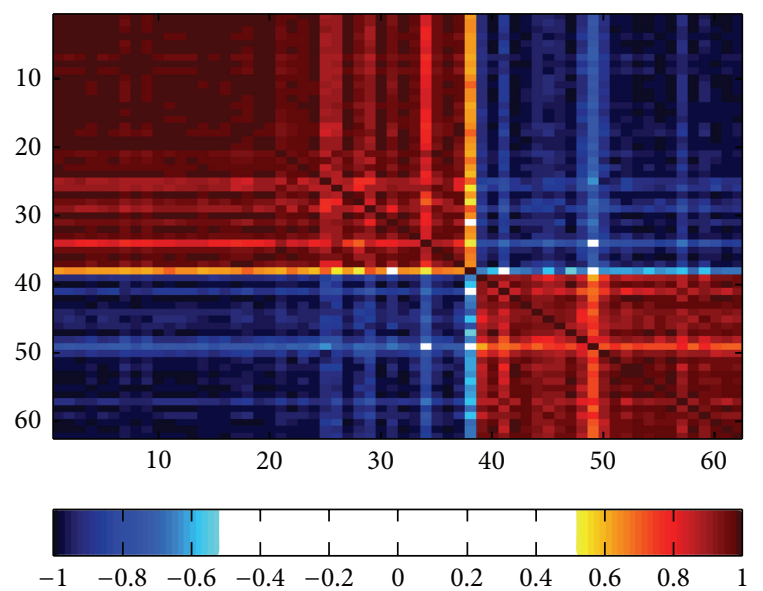

FIGURE 1: Gene correlation network of liver early development. $o b p 3$, extracellular region and transporter, was correlated to membrane (ust5r, stac3, cdh17, mme, olr59, gpm6a, tmem163, abcg8, abcd2, and $a b c c 3$ ), adapter (stac3, socs2), transcription (zfp 37, ccna2, asns, and rgd1562284), immune (rt1-ce5, rt1-aw2, cxcl13, and cyfip2), and redox (hsd3b5, cyp2c13, cyp2a2, dhrs7, hao2, akr1c1, nox4, inmt, dusp1, mettl7b, ppp2r2a, cyp17al, and $a k r 1 b 7)$. Genes were correlated using 2D STOCSY, $P<0.05$. Keys: 1,2 obp3; 3,4,7 rup2; 5, hsdsb5; 6, dhrs7; 8, cyp2c13; 16, ust5r; 19, stac3; 22, zfp37; 32, ppp2r2a; 33, socs2; 39, pcdh17; 41, abcg8; 44, ccna2; 49, s100g; 50, cxcl13; 53, tox; 62, akr1b7. The corresponding genes of numbers were listed in Supplementary Table 2.

ppp2r2a (Figure 2(b)), and obp3 expression inhibition $(0.05 \mu \mathrm{g} / \mathrm{mL}$ cycloheximide) downregulated $h s d 3 b 5$, socs 2 , pik3c3, and cav2 (Figure 2(c)).
3.8. Obps and Its Related Proteins. In order to characterize opb3 protein and its related proteins, we assayed Western blotting. The expression levels of obp3, hsd3b5, ppp2r2a, socs 2 , and pik3c3 proteins were elevated significantly in the adult rats compared to the pups, while the expression levels of cxcl13, tox, and akrlb7 proteins were decreased significantly (Figure 2(d), $P<0.01)$.

3.9. Metabolic Profile of Temporal Rats. Using PLS, invalid models indicated that adults were metabolically stationary from week 15 to week 19 of age (Table 4). With age (from week 8 to week 13 of age), taurine and octanoate (8:0) were increased, whilst succinate was lowered $(P<0.05$, Figure 3$)$.

\section{Discussions}

Early development is a physiology process, and we found that in this early individual development, extracellular region and space (ECR) - $\mathrm{SH}_{2}$ containing protein-MAPK pathway plays a vital role. Meanwhile, early individual development is ECR and androgen-mediated feedforward coordination network of positive cancer-like growth and negative regulations.

4.1. Androgen-Responsive Genes. Androgen-dependent $\alpha 2 \mathrm{u}$ globulin (obp3) is a group of low molecular weight $(\mathrm{Mr}$ $\sim 18,000$ ) male specific urinary proteins synthesized and secreted by hepatocytes. In the male rat, hepatic synthesis of $\alpha 2 \mathrm{u}$ globulin begins at puberty ( $\sim 40$ days), reaches a peak level ( $20 \mathrm{mg} /$ day $)$ at about 75 days, and declines during old age [21]. Age-dependent changes in the expression of androgen-responsive genes (alpha $2 \mathrm{u}$ globulin) reflect changing androgen sensitivity [2]. 


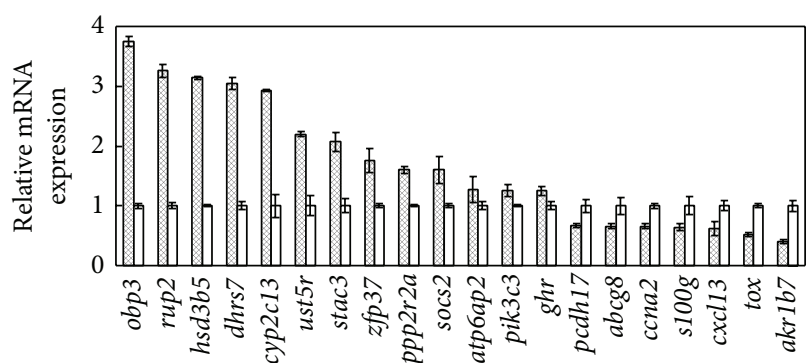

๑ Adult

口 Pup

(a)

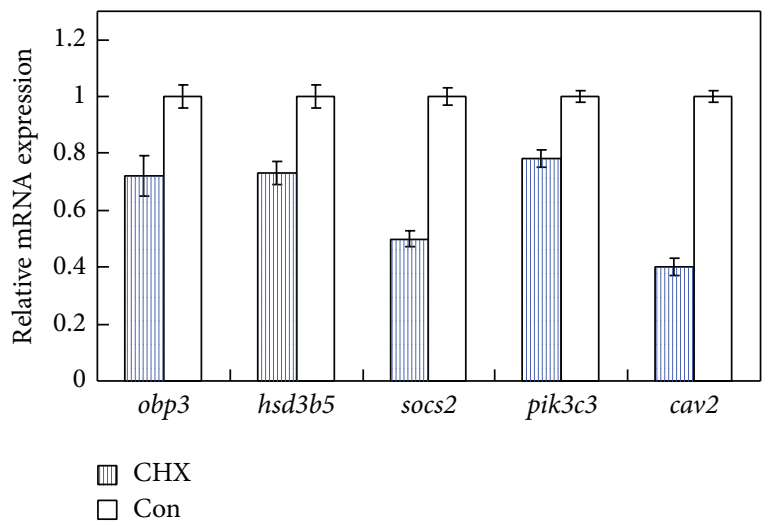

(c)

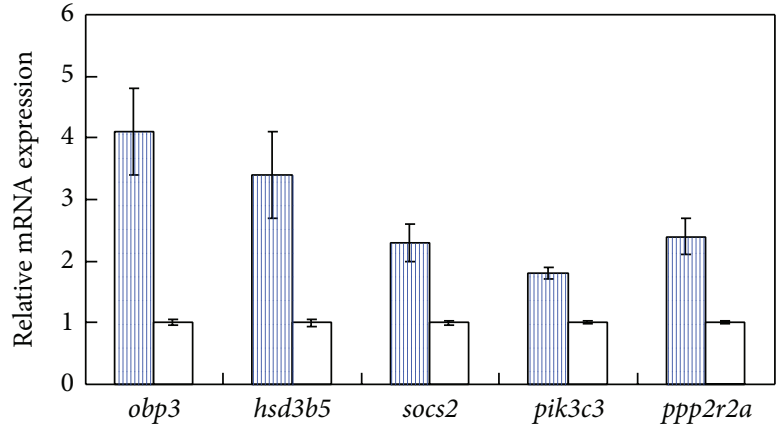

四 Dex

$\square$ Con

(b)

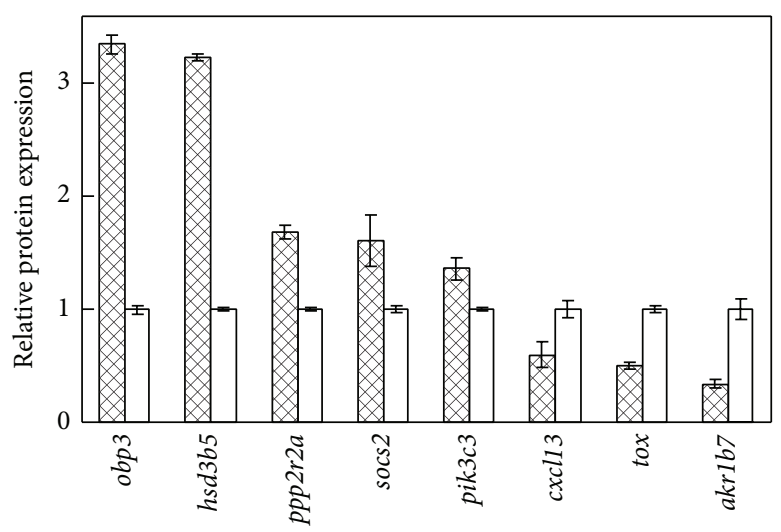

Adult

$\square$ Pup

(d)

FIGURE 2: mRNA and protein relative expression of liver early development. (a) qPCR validation for highlighted microarray genes between pups and adults, (b) $0.1 \mu \mathrm{M}$ dexamethasone (dex) induction of obp3 and coupregulation of its targets of pups, (c) $0.05 \mu \mathrm{g} / \mathrm{mL}$ cycloheximide ( $\mathrm{CHX}$ ) inhibition and codownregulation of related genes of pups, (d) Western blotting for key proteins between pups and adults. All genes/proteins are significant, $P<0.01$.

TABLE 4: PLS model parameters of temporal rats metabonome.

(a) Cumulative comparison

\begin{tabular}{lccccccc}
\hline \multirow{2}{*}{ Group } & & \multicolumn{3}{c}{$Q^{2}$, permutation test } & w19 & w21 & w23 \\
\hline Control & w10 & w13 & w15 & w17 & w19 & 0.790 & 0.885 \\
\hline
\end{tabular}

\#Comparison with w8.

(b) Link and other comparisons

\begin{tabular}{|c|c|c|c|c|c|c|c|c|}
\hline Group & $10-13$ & $13-15$ & $15-17$ & $17-19$ & $19-21$ & $21-23$ & & \\
\hline Control & 0.992 & 0.497 & 0.269 & 0.187 & 0.232 & 0.807 & & \\
\hline Group & $10-15$ & $10-17$ & $10-19$ & $10-21$ & $10-23$ & $13-17$ & $13-19$ & \\
\hline Control & 0.519 & 0.793 & 0.497 & 0.544 & 0.906 & 0.613 & 0.896 & \\
\hline Group & $13-21$ & $13-23$ & $15-19$ & $15-21$ & $15-23$ & $17-21$ & $17-23$ & $19-23$ \\
\hline Control & 0.677 & 0.919 & $<\mathbf{0}$ & 0.642 & 0.930 & 0.796 & 0.920 & 0.734 \\
\hline
\end{tabular}

Bold: invalid model. Components: autofit. 

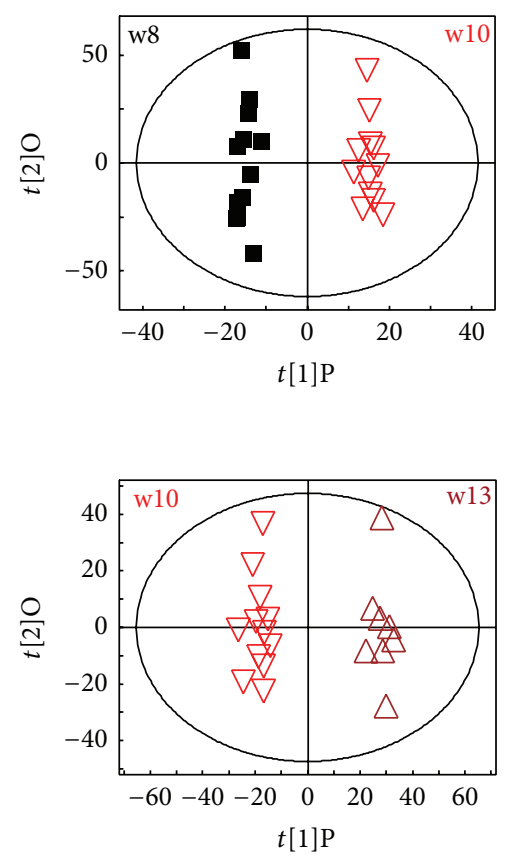

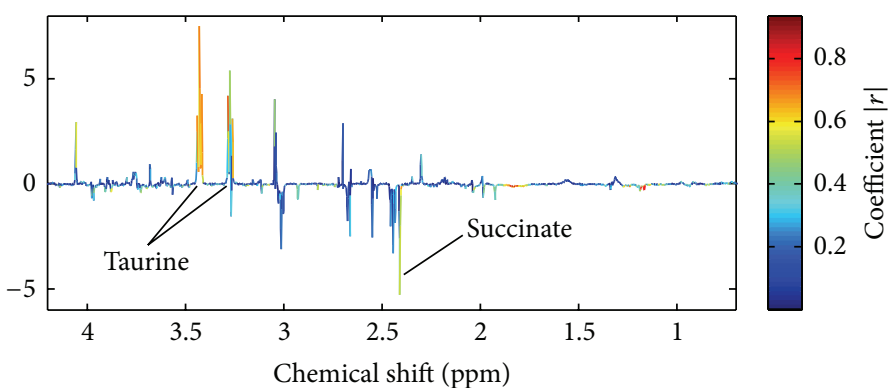

(a)

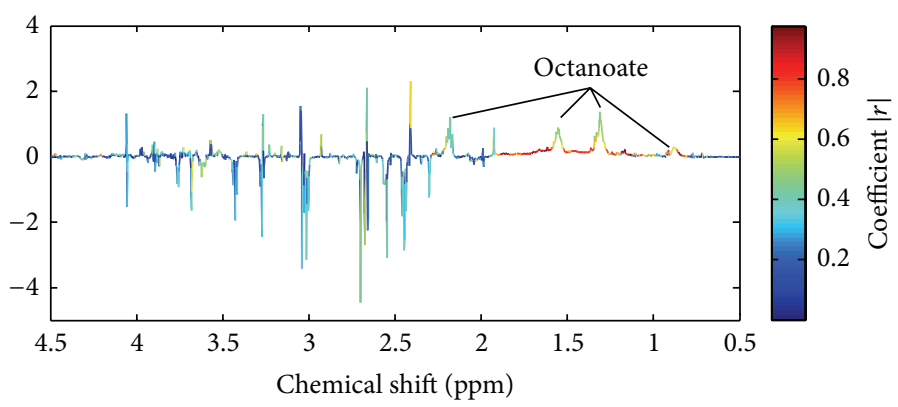

(b)

FIGURE 3: Taurine and octanoate increased with age. Cross validated qualitative O-PLS scores plots (left) and corresponding coefficient loading plots (right). Urine spectra comparison between w8 with w10 $\left(\mathrm{a}, Q^{2}=0.759\right)$ and w13 versus w10 $\left(\mathrm{b}, Q^{2}=0.992\right)$. Here, the red color indicated important discriminatory metabolites whereas the blue color indicated no significance in discrimination. w8: black boxes, w10: red inverted triangles, and w13: purple triangles.

Meanwhile, cell cycle and mitosis gene mapre1 at week 16 was upregulated 1.25 times than at week $3(P=0.0026)$.

Accordingly, androgen receptor at week 16 was upregulated 1.25 times than at week $3(P=2.4 E-05)$.

4.2. Development Network. Based on gene profiling, verification at mRNA, protein, and metabolite levels, we postulated that, in early development, extracellular region and space (ECR) obp3, rup2, pcdh17, a2m, and cxcl13 act as nutrition ligand and information input. Ligands interact with membrane transports ust5r, cdh17, mme, olr59, gpm6a, tmem163, abcg8, abcd2, abcc3, and $\mathrm{SH}_{2}$-containing/MAPK related signals stac3, socs2, cish/pik3c3, and $n r g 1$ and regulate cell cycle, transcription, and proteolysis ccng2, ccnb2, ccna2/zfp37, zfp68, npas2, taf9b, ppargcla, nfe2/hspb1, usp18, mup5, mme, trhde, spink3, and prcp, leading to short-term steroid, fatty acid biosynthesis, redox, and metabolic process obp3, hsd3b5, akr1c1, hsdl2, hsd17b6, hsd11b2, ar, cyp17a1/scd, fasn/cyp2c13, cyp3a9, cyp2a2, hao2, cyp2c29, nox4, me3, cyp2c12, akr1b7/dhrs7, mett17b, asns, acsm2, psat1 and longterm collagen development and organization col4a1, col5a2, col5a1, colla2, colla1/cav2, pex11a, onecut1, meox2, cml3, and lox. G protein coupled receptors/G protein olr59, rgs3 [22], adora2b/gnai3, gnat3, catalytic receptors socs2 [23], nim1 [24], atp6ap2, ghr and ECR signals converge at MAPK cascades (Figure 4). Protein expression to some extent confirmed key genes, for example, obp3, socs2, ppp2r2a, pik3c3, cxcl13, and hsd3b5 proteins dynamics (Figure 2).
4.3. Female-Prefer Genes. Female-specific tox changes in gene expression during postnatal liver development reflect the deceleration of liver growth and the induction of specialized liver functions, with widespread changes in sex-specific gene expression primarily occurring in male liver [25].

Male and female genes are both increased, but their magnitudes in male are larger than that in female-change fold of male gene $o b p 3$ is 3.75 times more at senior than at junior.

4.4. Development and Cancer. Hsd3b5 expression showed significant associations with the degree of hepatic steatosis [26], accompanied by increased testosterone with age (Table 1). Expression level of dehydrogenase/reductase member 7 (dhrs7) in rat regenerating liver was more than 968fold compared to control [27]. Cdh17 [28], nim1 [24], scd, and fasn [29] were related to disease/cancer; upregulation of fasn was in accordance with elevated moderate-chain fatty acid octanoate $(8: 0)$. Thus, developmental process poses cancerlike characteristics.

Overlap between embryonic liver development and liver cancer is not only in cell cycle or apoptosis, but also in metabolic pathways associated with carbohydrate and lipid metabolism [30]. Fetal hepatocytes have high IGF2 and E2F3 expressions, and levels of IGF2 and E2F3 mRNA were positively correlated to human prostate and bladder cancers [31]. However, fetal and infant livers have no cancers.

4.5. Negative Control Genes. Socs2 [23], cdkn1a [32], rgs3 [22], cish, spink3, cyp17a1, and $n f e 2$ [33] were involved in negative 


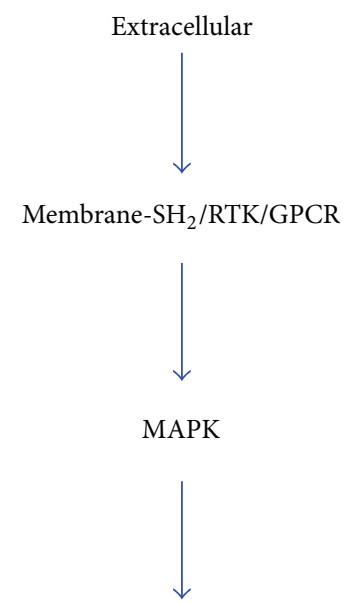

Transcription

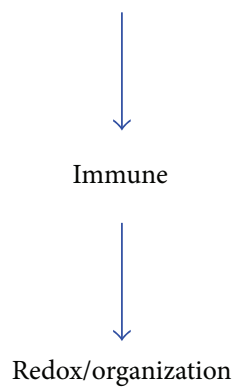

FIGURE 4: ECR-MAPK-mediated early individual development network. Extracellular region and space (ECR) act as nutrition ligand and information input. Ligands interact with membrane transports and $\mathrm{SH}_{2}$-containing/MAPK related signals and regulate cell cycle, transcription, and proteolysis, leading to short-term steroid, fatty acid biosynthesis, redox and metabolic process, and long-term collagen development and organization. $G$ protein coupled receptors/G protein, catalytic receptors, and ECR signals converge at MAPK cascades.

control. Cancer-like early individual development, but no cancer, is maybe due to counteracting effects of negative control and cooperation of the two sides.

4.6. Feedforward Regulation. Feedforward regulation in pheromone-activated MAPK pathway ensures stability and rapid reversibility of a cellular state [34].

Cxcl13, belonging to extracellular region [35], takes part in positive regulation of cytosolic calcium ion concentration and immune response [36].

Nonzero uterus dependent initial conditions allow fast early development and sensing, and meanwhile, feedforward modulations appear at reversible developmental transitions, because this network control can obtain the aims of growth stability and rapid reversibility without loss of external signaling information [34].

In a summary, $\mathrm{qPCR}$ validation was for gene expression profile, and meanwhile, cell induction/inhibition assays, Western blot, and NMR-based metabonomics were carried out for confirmation of gene results. Using dynamic assays of body weight, serum biochemistry, transcript, protein, and metabolite profile, we reveal that, in early individual development, increasing magnitude in male is larger than that in female, and cancer-like growth coordinates negative regulation; meanwhile, feedforward modulations appear at developmental transitions, obtaining aims of growth stability and rapid reversibility without superoxidation or maglinant growth; more importantly, extracellular matrix-kinase cascade responses play a vital role in this early individual development. Taken together, extracellular matrix-kinase cascadebased feedforward cooperation of cancer-like growth and negative regulation realize win-win long-term growth stability and short-term rapid reversibility/fluctuation in gradual transition of early individual development. This finding is particularly important for understanding the gene expression network of infant development.

\section{Conflict of Interests}

The authors declare that there is no conflict of interests regarding the publication of this paper.

\section{Authors' Contribution}

Xiu-Ju Zhao conceived of the study, designed the analysis, coordinated the analyses and interpretation of results, and drafted the paper; Hexian Zhuo participated in the design of the study. All authors read, edited, and approved the final paper.

\section{Acknowledgments}

The authors would like to acknowledge KR Shockley. This work was supported in part by Natural Science Foundation of Hubei Province (2012FFB04802), Wuhan Polytechnic University (2012RZ06).

\section{References}

[1] A. K. Roy and B. Chatterjee, "Sexual dimorphism in the liver.," Annual Review of Physiology, vol. 45, pp. 37-50, 1983.

[2] B. Chatterjee, G. Fernandes, B. P. Yu et al., "Calorie restriction delays age-dependent loss in androgen responsiveness of the rat liver," FASEB Journal, vol. 3, no. 2, pp. 169-173, 1989.

[3] B. T. Spear, L. Jin, S. Ramasamy, and A. Dobierzewska, “Transcriptional control in the mammalian liver: liver development, perinatal repression, and zonal gene regulation," Cellular and Molecular Life Sciences, vol. 63, no. 24, pp. 2922-2938, 2006.

[4] T. Li, J. Huang, Y. Jiang et al., "Multi-stage analysis of gene expression and transcription regulation in C57/B6 mouse liver development," Genomics, vol. 93, no. 3, pp. 235-242, 2009.

[5] J. K. Dunnick, A. Brix, H. Cunny, M. Vallant, and K. R. Shockley, "Characterization of polybrominated diphenyl ether toxicity in wistar han rats and use of liver microarray data for predicting disease susceptibilities," Toxicologic Pathology, vol. 40, no. 1, pp. 93-106, 2012.

[6] V. G. Tusher, R. Tibshirani, and G. Chu, "Significance analysis of microarrays applied to the ionizing radiation response," 
Proceedings of the National Academy of Sciences of the United States of America, vol. 98, no. 9, pp. 5116-5121, 2001.

[7] J. D. Storey and R. Tibshirani, "Statistical significance for genomewide studies," Proceedings of the National Academy of Sciences of the United States of America, vol. 100, no. 16, pp. 9440-9445, 2003.

[8] C.-A. Tsai, H.-M. Hsueh, and J. J. Chen, "Estimation of false discovery rates in multiple testing: application to gene microarray data," Biometrics, vol. 59, no. 4, pp. 1071-1081, 2003.

[9] V. Todorov and P. Filzmoser, "Robust statistic for the one-way MANOVA," Computational Statistics and Data Analysis, vol. 54, no. 1, pp. 37-48, 2010.

[10] M. W. Pfaffl, "A new mathematical model for relative quantification in real-time RT-PCR," Nucleic Acids Research, vol. 29, no. 9, p. e45, 2001.

[11] W. P. Aue, E. Bartholdi, and R. R. Ernst, "Two-dimensional spectroscopy. Application to nuclear magnetic resonance," The Journal of Chemical Physics, vol. 64, no. 5, pp. 2229-2246, 1976.

[12] W. P. Aue, J. Karhan, and R. R. Ernst, "Homonuclear broad band decoupling and two-dimensional J-resolved NMR spectroscopy," Journal of Chemical Physics, vol. 64, no. 10, pp. 42264227, 1976.

[13] L. Braunschweiler and R. R. Ernst, "Coherence transfer by isotropic mixing: application to proton correlation spectroscopy," Journal of Magnetic Resonance, vol. 53, no. 3, pp. 521528, 1983.

[14] X.-J. Zhao, C. Huang, H. Lei, X. Nie, H. Tang, and Y. Wang, "Dynamic metabolic response of mice to acute mequindox exposure," Journal of Proteome Research, vol. 10, no. 11, pp. 51835190, 2011.

[15] J. Trygg and S. Wold, "Orthogonal projections to latent structures (O-PLS)," Journal of Chemometrics, vol. 16, no. 3, pp. 119$128,2002$.

[16] C. Henegar, K. Clement, and J. Zucker, "Unsupervised multipleinstance learning for functional profiling of genomic data," in Proceedings of the European Conference on Machine Learning and Principles and Practice of Knowledge Discovery in Databases, pp. 186-197, 2006.

[17] R. Opgen-Rhein and K. Strimmer, "From correlation to causation networks: a simple approximate learning algorithm and its application to high-dimensional plant gene expression data," BMC Systems Biology, vol. 1, article 37, 2007.

[18] T. Hastie, R. Tibshirani, and J. Friedman, The Elements of Statical Learning, Springer, New York, NY, USA, 2nd edition, 2008.

[19] F. Lindgren, B. Hansen, W. Karcher, M. Sjöström, and L. Eriksson, "Model validation by permutation tests: applications to variable selection," Journal of Chemometrics, vol. 10, no. 5-6, pp. 521-532, 1996.

[20] E. Axelsson, A. Ratnakumar, M.-L. Arendt et al., “The genomic signature of dog domestication reveals adaptation to a starchrich diet," Nature, vol. 495, no. 7441, pp. 360-364, 2013.

[21] A. K. Roy, B. Chatterjee, K. V. S. Rao, C. V. R. Murty, F. H. Sarkar, and D. Majumdar, "Androgenic regulation of hepatic gene expression," The Journal of Steroid Biochemistry, vol. 27, no. 4-6, pp. 1129-1134, 1987.

[22] Q. Wang, M. Liu, B. Mullah, D. P. Siderovski, and R. R. Neubig, "Receptor-selective effects of endogenous RGS3 and RGS5 to regulate mitogen-activated protein kinase activation in rat vascular smooth muscle cells," Journal of Biological Chemistry, vol. 277, no. 28, pp. 24949-24958, 2002.
[23] B. R. Dey, S. L. Spence, P. Nissley, and R. W. Furlanetto, "Interaction of human suppressor of cytokine signaling (SOCS)-2 with the insulin-like growth factor-I receptor," The Journal of Biological Chemistry, vol. 273, no. 37, pp. 24095-24101, 1998.

[24] T. M. P. Team, "The status, quality, and expansion of the NIH full-length cDNA project: the Mammalian Gene Collection (MGC)," Genome Research, vol. 14, pp. 2121-2127, 2004.

[25] T. Conforto and D. Waxman, "Sex-specific mouse liver gene expression: genome-wide analysis of developmental changes from pre-pubertal period to young adulthood," Biology of Sex Differences, vol. 3, p. 9, 2012.

[26] N. Guillén, M. A. Navarro, C. Arnal et al., "Microarray analysis of hepatic gene expression identifies new genes involved in steatotic liver," Physiological Genomics, vol. 37, no. 3, pp. 187-198, 2009.

[27] W.-B. Wang, J.-M. Fan, X.-L. Zhang, J. Xu, and W. Yao, "Serial expression analysis of liver regeneration-related genes in rat regenerating liver," Molecular Biotechnology, vol. 43, no. 3, pp. 221-231, 2009.

[28] J. Wang, W.-M. Kang, J.-C. Yu, Y.-Q. Liu, Q.-B. Meng, and Z.-J. Cao, "Cadherin-17 induces tumorigenesis and lymphatic metastasis in gastric cancer through activation of NF $\kappa \mathrm{B}$ signaling pathway," Cancer Biology and Therapy, vol. 14, no. 3, pp. 262270, 2013.

[29] S. B. Biddinger, K. Almind, M. Miyazaki, E. Kokkotou, J. M. Ntambi, and C. R. Kahn, "Effects of diet and genetic background on sterol regulatory element-binding protein-1c, stearoyl-CoA desaturase 1, and the development of the metabolic syndrome," Diabetes, vol. 54, no. 5, pp. 1314-1323, 2005.

[30] D. Becker, I. Sfakianakis, M. Krupp et al., "Genetic signatures shared in embryonic liver development and liver cancer define prognostically relevant subgroups in HCC," Molecular Cancer, vol. 11, article 55, 2012.

[31] J. C. Lui and J. Baron, "Evidence that Igf2 down-regulation in postnatal tissues and up-regulation in malignancies is driven by transcription factor E2f3," Proceedings of the National Academy of Sciences of the United States of America, vol. 110, no. 15, pp. 6181-6186, 2013.

[32] F. C. C. Rodrigues, R. S. Kawasaki-Oyama, J. F. G. Fo et al., "Analysis of CDKN1A polymorphisms: markers of cancer susceptibility?" Cancer Genetics and Cytogenetics, vol. 142, no. 2, pp. 92-98, 2003.

[33] J. Wang, K. Huo, L. Ma et al., "Toward an understanding of the protein interaction network of the human liver," Molecular Systems Biology, vol. 7, article 536, 2011.

[34] A. Doncic and J. M. Skotheim, "Feedforward regulation ensures stability and rapid reversibility of a cellular state," Molecular Cell, vol. 50, no. 6, pp. 856-868, 2013.

[35] T. A. Rupprecht, C. J. Kirschning, B. Popp et al., "Borrelia garinii induces CXCL13 production in human monocytes through tolllike receptor 2," Infection and Immunity, vol. 75, no. 9, pp. 43514356, 2007.

[36] C.-H. Jenh, M. A. Cox, W. Hipkin et al., "Human B cellattracting chemokine 1 (BCA-1; CXCL13) is an agonist for the human CXCR3 receptor," Cytokine, vol. 15, no. 3, pp. 113-121, 2001. 

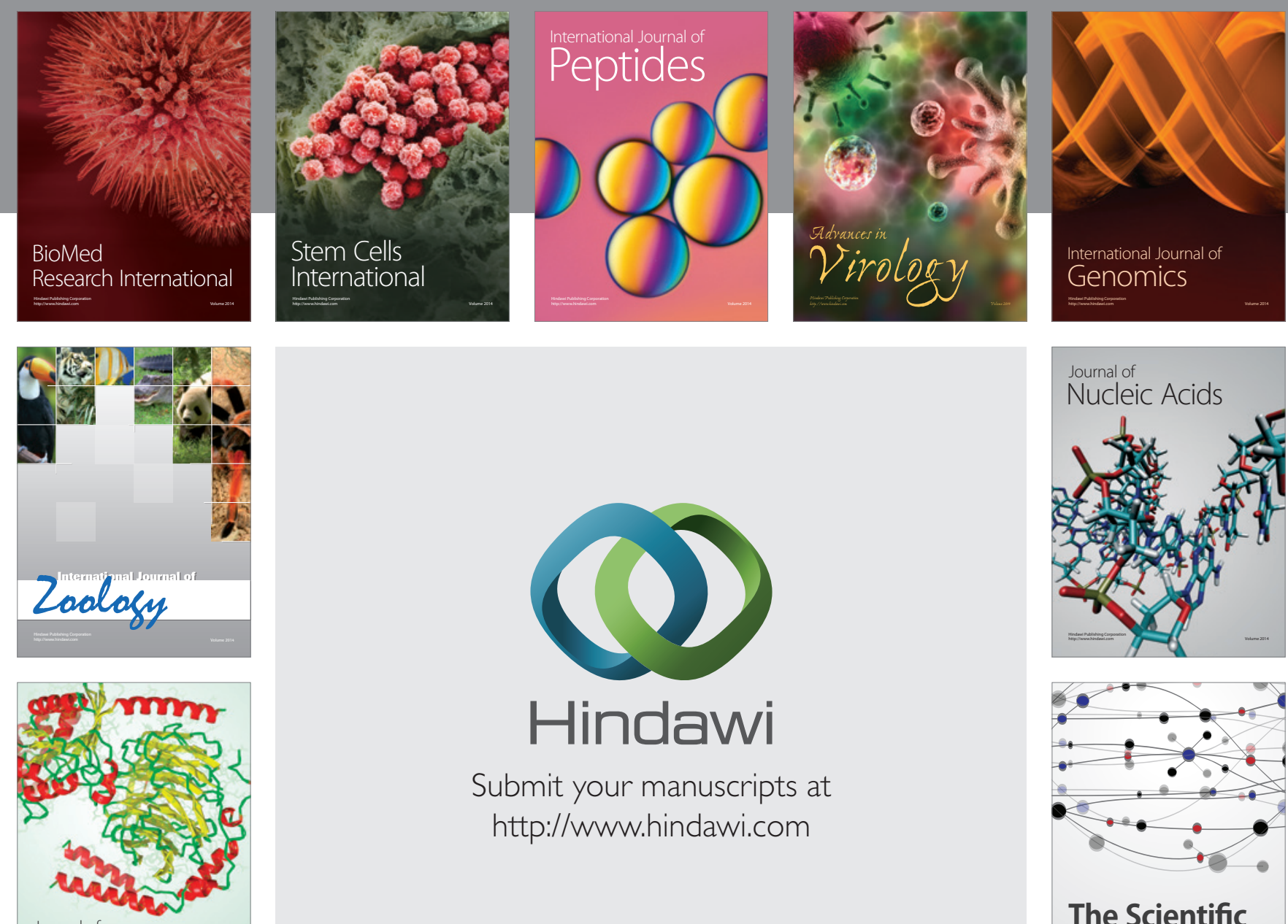

Submit your manuscripts at

http://www.hindawi.com

Journal of
Signal Transduction
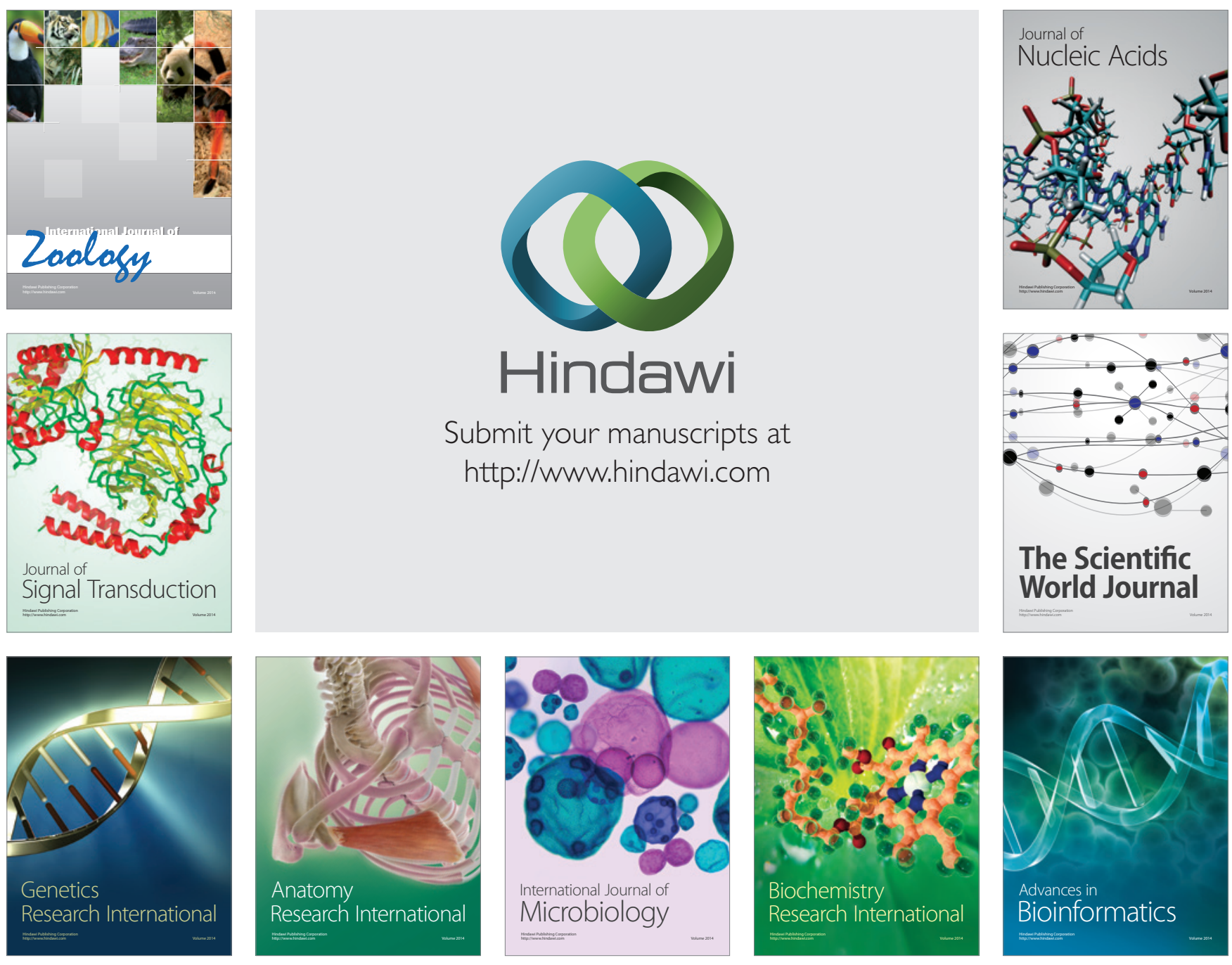

The Scientific World Journal
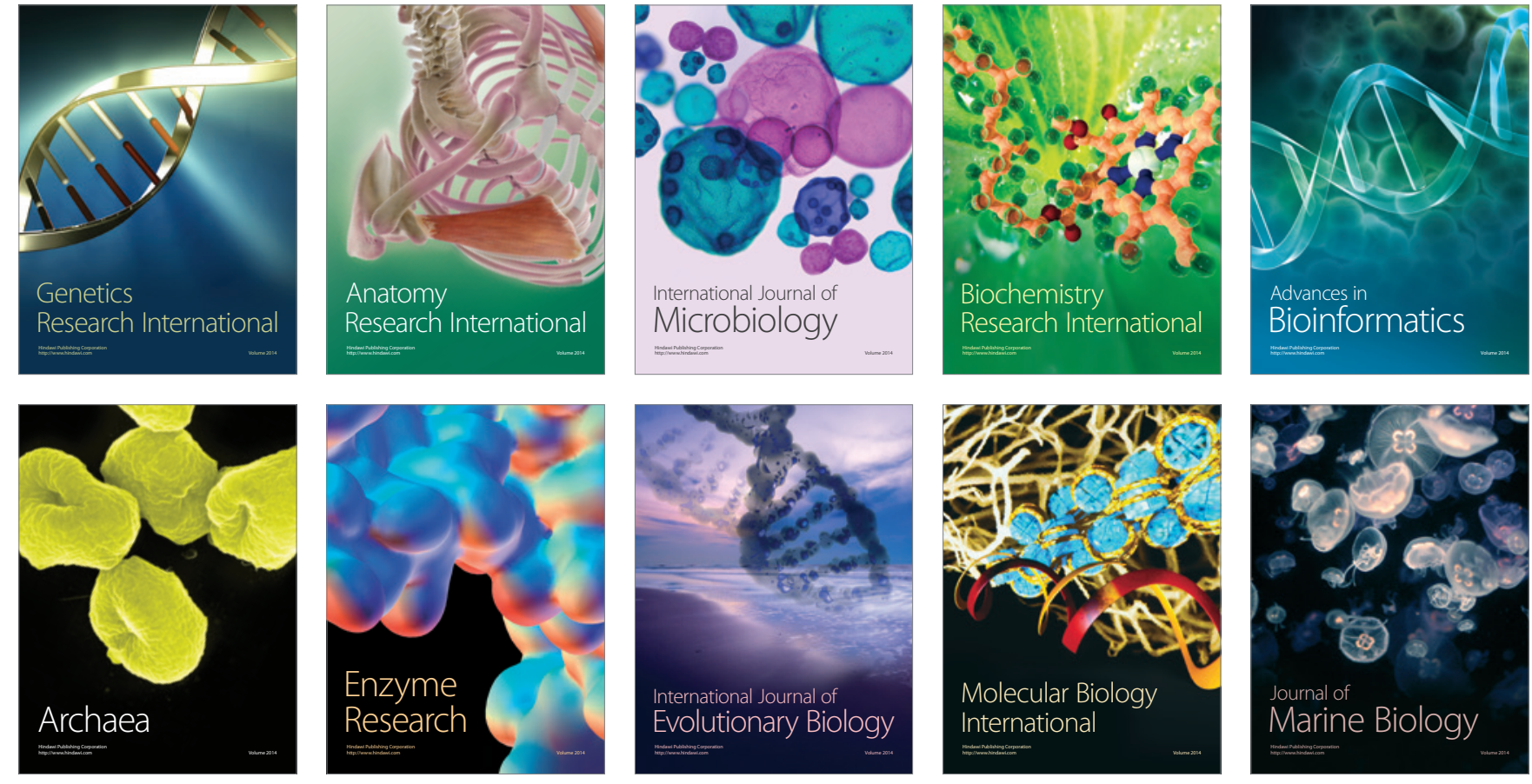